\title{
Keratoprosthesis in the fight against corneal blindness in developing countries
}

\author{
Ceratoprótese na luta contra cegueira corneana em países em desenvolvimento
}

Lauro Oliveira ${ }^{1}$, Fabiano Cade $^{2}$, Claes Dohlman ${ }^{3}$

${ }^{1}$ Department of Ophthalmology, São Paulo Federal University - UNIFESP São Paulo, Brazil.

2 Department of Ophthalmology, São Paulo Federal University - UNIFESP São Paulo, Brazil.

${ }_{3}^{3}$ Masscahusetts Eye and Ear Infirmary, Harvard Medical School, Boston, MA, USA.
W hy should we attempt to develop keratoprostheses (KPros) for corneal blindness when standard corneal transplantation is so well established and relatively safe? It is true that penetrating keratoplasty, or variations thereof such as endothelial keratoplasty or deep anterior lamellar keratoplasty, are often effective measures in the surgical management of corneal opacification from various causes. However, there are at least two reasons why we need a viable alternative beyond such standard measures. One is due to widespread shortage of donor material and health resources in general, and the other - more important - is the failure rate of keratoplasty in severe cases. Thus we need a back up procedure that can succeed when keratoplasty has failed - and particularly so in the Developing World with its high incidence of corneal disease.

According to recent studies, the World Health Organization (WHO) estimates that the global number of people who are considered blind is 39 million (1). Diseases affecting the cornea constitute a major cause of blindness worldwide, second only to cataract in overall importance ${ }^{(2)}$. In low-income Developing Countries, there is a disproportional prevalence of corneal disease as a major cause of blindness. Its epidemiology varies from country to country, depending on many factors, such as the lack of resources for prevention and availability of eye care services ${ }^{(3)}$. For rehabilitation only about a hundred thousand standard corneal transplants are performed worldwide per year at present ${ }^{(4)}$, which is not enough for the demand. The insufficient supply of corneal donor tissue for keratoplasty as well as insufficient eye bank organizations, contribute to explain the high global burden of visual disability due to untreated corneal diseases. The other important issue refers to long term outcome with standard keratoplasty which is more limited than often believed. It varies markedly with underlying disease, geography, availability of donor tissue, health budget, and other factors, and it is still poorly documented. Most of the outcome studies published is not population-based and they usually deal with cases of good prognosis in the Developed World. There are situations where the prognosis for successful keratoplasty is very poor, such as in multiple graft failures, severely vascularized corneas, stem cell deficiencies, autoimmune diseases, severe chemical burns, etc ${ }^{(5)}$.

Historically, Keratoprosthesis designs have been beset by technical challenges and by a significant incidence of post-operative complications. The heterogeneity of the data on the different devices, a lack of prospective evaluations, and small numbers of procedures have raised concerns about safety, KPro survival rates, and associated complications.

The Boston Type I Keratoprosthesis has become the most common procedure for cases where standard keratoplasty has failed ${ }^{(6)}$. Multicenter and medium term outcome studies have been encouraging ${ }^{(7)}$. This Keratoprosthesis is now used at a rate of about 1,200 per year, worldwide. The Type I consists of two polymethyl methacrylate plates sandwiching a corneal autograft or allograft, which is then implanted like a standard graft, single stage. A soft contact lens is permanently used after surgery to prevent corneal dehydration and to minimize the risk of melting of the carrier cornea ${ }^{(8)}$. Life-long prophylaxis with lowdose topical antibiotics (e.g. one drop daily) is necessary. Long-term complications associated with the device include glaucoma, retroprosthetic membrane formation, sterile vitritis, retinal detachment and, now rarely, endophthalmitis(9). A Type II device with an extended nub that protrudes a closed lid is occasionally used in end-stage dry eyes(6).

Boston Keratoprosthesis implantation has been proven to be a highly cost-effective medical intervention in the US ${ }^{(10)}$. This procedure, even as a first option in selected patients, can decrease health treatment costs. In contrast, penetrating keratoplasty e.g. in Africa has a limited role and often poor results in infectious and inflammatory diseases, which are the main causes of corneal blindness in Developing Countries ${ }^{(11)}$. This relatively limited ability of standard keratoplasty in restoring sight to blind patients will undoubtedly tilt the choices more and more in favor of KPro.

Research on keratoprostheses is currently vigorous and is directed to problems such as inflammatory response in autoimmune diseases and chemical burns, infections, new drug-eluting contact lenses, optics, glaucoma, shunts, etc $\mathrm{c}^{(12-16)}$. Undoubtedly there will be rapid technological expansion in this field but will the underlying economics in the Developing World keep up with the pace? The total cost is not so much due to the cost of the device but rather physician time, patient travel, antibiotics, contact lenses and education. Still, with the rapidly expanding economies of the Developing Nations, there is great likelihood that the Keratoprosthesis option will be more and more utilized. Keratoprostheses will undoubtedly become more affordable but they will also - most importantly - be safer. 


\section{REFERENCES}

1. World Health Organization. WHO release the new global estimates on visual impairment. Geneva:WHO; 2010. [cited 2011 March 23]. Available from: http://www.who.int/blindness/en

2. Whitcher JP, Srinivasan M, Upadhyay MP. Corneal blindness: a global perspective. Bull World Health Organ. 2001;79(3):214-21.

3. Smith GT, Taylor HR. Epidemiology of corneal blindness in developing countries. Refract Corneal Surg. 1991;7(6):436-9. Comment in: Refract Corneal Surg. 1991;7(6):417-8.

4. Steve Cole. Eye Bank Association of America. Washington: DC; 2009. [personal communication].

5. Maguire MG, Stark WJ, Gottsch JD, Stulting RD, Sugar A, Fink NE, et al. Risk factors for corneal graft failure and rejection in the collaborative corneal transplantation studies. Collaborative Corneal Transplantation Studies Research Group. Ophthalmology. 1994; 101(9):1536-47.

6. Dohlman CH, Harrisi-Dagher M, Khan BF, Sippel K, Aquavella JV, Graney JM. Introduction to the use of the Boston keratoprosthesis. Expert Rev Ophthalmol 2006;1 (1):41-8.

7. Zerbe BL, Belin MW, Ciolino JB; Boston Type 1 Keratoprosthesis Study Group. Results from the multicenter Boston Type 1 Keratoprosthesis Study. Ophthalmology. 2006;113(10): 1779e1-7.
8. Dohlman C, Dudenhoefer E, Khan BF, Morneault S. Protection of the ocular surface after keratoprosthesis surgery: the role of soft contact lenses. CLAO J. 2002;28(2):72-4.

9. Aldave AJ, Kamal KM, Vo RC, Yu F. The Boston type I keratoprosthesis: improving outcomes and expanding indications. Ophthalmology. 2009;116(4):640-51.

10. Ament JD, Stryjewski TP, Ciolino JB, Todani A, Chodosh J, Dohlman CH. Cost-effectiveness of the Boston Keratoprosthesis. Am J Ophthalmol. 2010;149(2):221-8.e.2.

11. Yorston D, Wood M, Foster A. Penetrating keratoplasty in Africa: graft survival and visual outcome. Br J Ophthalmol. 1996;80(10):890-4.

12. Ciralsky J, Papaliodis GN, Foster CS, Dohlman CH, Chodosh J. Keratoprosthesis in autoimmune disease. Ocul Immunol Inflamm. 2010;18(4):275-80.

13. Durand ML, Dohlman CH. Successful prevention of bacterial endophthalmitis in eyes with the Boston keratoprosthesis. Cornea. 2009;28(8):896-901.

14. Ciolino JB, Hoare TR, Iwata NG, Behlau I, Dohlman CH, Langer R, et al. A drug-eluting contact lens. Invest Ophthalmol Vis Sci. 2009;50(7):3346-52.

15. Sayegh RR, Avena Diaz L, Vargas-Martin F, Webb RH, Dohlman CH, Peli E. Optical functional properties of the Boston Keratoprosthesis. Invest Ophthalmol Vis Sci. 2010; 51(2):857-63.

16. Rivier D, Paula JS, Kim E, Dohlman CH, Grosskreutz CL. Glaucoma and keratoprosthesis surgery: role of adjunctive cyclophotocoagulation. J Glaucoma. 2009;18(4):321-4. 